\title{
Der TARMED wird noch nicht überall kostenneutral angewendet!
}

\author{
3. Sitzung KN-Büro vom 19. August 2004
}

U. Stoffel, C. Favrod-Coune

1 Stoffel U, Favrod-Coune C. Die Kostenneutralität wird gelebt, aber nicht überall! Schweiz Ärztezeitung 2004;85(29/30):1525.
Korrespondenz:

Backoffice KN-Büro G7

Pflanzschulstrasse 3

CH-8400 Winterthur

Tel. 0522351935

Fax 0522351920

E-Mail: KNBueroG7@hin.ch
Das KN-Büro hat am 19. August 2004 erste Reduktionen, aber auch Erhöhungen der Taxpunktwerte ab 1. September 2004 beschlossen.

Die dritte Sitzung des KN-Büros hat am 19. August 2004 stattgefunden. Die Delegation der G7 (Dres. U. Stoffel und C. Favrod-Coune) konnte sich auf die Daten aus den TrustCenter abstützen. Diese umfassen mittlerweile bereits über 3 Mio. TARMED-Rechnungen aus fast 5000 Praxen in der ganzen Schweiz.

Die vom Backoffice des KN-Büros G7 (NewIndex) aufbereiteten Analysen und Statistiken wurden wiederum den Messresultaten von santésuisse gegenübergestellt, um in allen KN-Räumen Klarheit über die Einhaltung der Kostenneutralität zu schaffen.

Das Fazit der 3. KN-Sitzung kann wie folgt zusammengefasst werden:

- Die Entwicklung der Kosten der freipraktizierenden Ärztinnen und Ärzte verläuft gesamtschweizerisch noch im vorgegebenen KN-Korridor. Die Abweichung von der Vorgabe beträgt im Juni 2004 knapp 2\% und liegt damit im Rahmen der Erwartungen.

- In insgesamt sieben KN-Räumen mussten die Taxpunktwerte reduziert werden. In zwei KN-Regionen konnte der Taxpunktwert um 4 bzw. 6 Rappen erhöht werden. Die Reduktionen bewegen sich zwischen 2 und 6 Rap- pen. Dank der ausgezeichneten eigenen Daten konnte das Ausmass der Reduktionen mehrheitlich in Grenzen gehalten werden. Die Beschlüsse erfolgten im Rahmen der Vorgaben des KN-Vertrages und im Konsens aller Parteien.

- Die Delegation der G7 hat im KN-Büro - aufgrund der erheblichen Abweichungen in den Abrechnungsvolumen der Versicherer im 1. und 2. Quartal 2004 - Fragen über die quantitativen Auswirkungen der Tarifumstellung auf die Messung der Kostenneutralität deponiert. Diese Fragen sind vom KNBüro aufgenommen worden und haben kurzfristig zu einer zurückhaltenden Anpassung der Taxpunktwerte beigetragen. Auf die kommende Sitzung des KN-Büros werden die Auswirkungen dieser Volumenverschiebungen gegenüber der erwarteten «Saisonalität» weiter abgeklärt.

Wie bereits im letzten Info [1] angezeigt, ist der Spielraum, in dem wir uns bewegen müssen, sehr eng bemessen. Dies erfordert von allen Beteiligten äusserste Disziplin in der TARMED-Anwendung! Diese muss in allen KN-Räumen gemäss der «politischen» Vorgaben der Kostenneutralität erfolgen. Nur so kann eine Erosion der Taxpunktwerte vermieden werden.

Tabelle 1

Taxpunktwerte ab 1. September 2004. Stand nach den Beschlüssen des KN-Büros vom 19. August 2004.

\begin{tabular}{llll} 
Kanton/KN-Raum & Initialwert (in Rp.) & Neu ab 1.9.2004 (in Rp.) & Korrektur (in Rp.) \\
\hline Zürich & 97 & 95 & -2 \\
\hline Bern & 89 & 86 & -3 \\
\hline Fribourg & 91 & 91 & - \\
\hline Solothurn & 87 & 84 & -3 \\
\hline Basel-Stadt & 93 & 93 & - \\
\hline Baselland & 97 & 97 & - \\
\hline Graubünden & 84 & 78 & -6 \\
\hline Aargau & 89 & 89 & - \\
\hline Tessin & 91 & 97 & 6 \\
Waadt & 92 & 96 & 4 \\
\hline Wallis & 78 & 78 & - \\
\hline Neuenburg & 94 & 89 & -5 \\
\hline Genf & 98 & 98 & - \\
\hline Jura & 95 & 95 & - \\
Zentral-CH & 86 & 83 & -3 \\
Ost-CH & 85 & 82 & -3
\end{tabular}

